\title{
光照条件下碳-硅键断裂及其溴代反应研究
}

\author{
孙京 $a$ 彭新华*,a 郭 浩*,b \\ ( ${ }^{a}$ 南京理工大学化工学院 南京 210094) \\ $\left({ }^{b}\right.$ 复旦大学化学系 上海 200433)
}

\begin{abstract}
摘要 研究了在高能量紫外光照条件下, 芐基硅试剂的碳一硅键断裂过程及随后的高效澳代反应. 该反应在紫外光照 条件下，室温下即可温和且高效地进行，从而发展了一套新型的由芐基硅试剂合成芐基溴代物的方法. 同时提出了该 反应的可能机理.

关键词 光化学; 澳代反应; 机理; 自由基反应; 有机硅试剂
\end{abstract}

\section{Studies on Photoinduced Carbon-Silicon Bond Cleavage and Subsequent Bromination Reaction}

\author{
Sun, Jing ${ }^{a}$ Peng, Xinhua ${ }^{*, a}$ Guo, Hao ${ }^{*, b}$ \\ ( ${ }^{a}$ School of Chemical Engineering, Nanjing University of Science and Technology, Nanjing 210094) \\ ( ${ }^{b}$ Department of Chemistry, Fudan University, Shanghai 200433)
}

\begin{abstract}
A novel photoinduced bromination of benzylsilanes is reported. This reaction undergoes smoothly under UV irradiation at room temperature via two key steps: $\mathrm{C}-\mathrm{Si}$ bond cleavage and subsequent bromination, which presents a new protocol to the synthesis of benzyl bromides. In addition, a possible mechanism of this reaction is proposed.

Keywords photochemistry; bromination; mechanism; radical reactions; organicsilanes
\end{abstract}

苄基卤代物作为合成医药、农药、染料、香料的重 要原料而被广泛应用 ${ }^{[1 \sim 4]}$. 同时, 它也是合成各种精细 化学品和生物活性化合物的常用中间体 ${ }^{\left[{ }^{5}\right.}$. . 发展高效 合成苄基溴代物的方法无论是在有机合成中还是在工 业生产过程中都是一项重要的研究课题 ${ }^{[9 \sim 12]}$.

到目前为止, 从有机硅试剂出发, 经过溴代反应过 程, 而高效生成相应的溴代物的方法报道还比较少 $见^{[13,14]}$. 这也许是因为从比较昂贵的有机硅试剂出发来 合成相对廉价的澳代物并不经济. 然而从合成方法学的 角度讲, 发展高效的有机硅试剂的澳代反应是对合成方 法学的有益补充. 从事此类反应的研究, 有助于推动有 机化学的发展. 我们小组在之前的研究工作中发展了一 套澳试剂催化的苄基硅试剂在光照条件下的脱硅及氧 化反应 ${ }^{[15]}$. 在此类反应中, 澳原子参与的单电子转移促 进的碳一硅键断裂是一个关键步骤. 为了扩展此类反应, 我们小组又进行了其它研究. 在这里我们将报道: 有机
硅试剂在光照条件下高效转化为相应芐溴类化合物的 方法.

\section{1 结果与讨论}

\section{1 反应条件优化}

首先，我们选取 4-甲氧基茮基三甲基硅烷(1a)作为 标准反应底物, $N$-溴代丁二酰亚胺(NBS)作为溴源, 考 察了溶剂效应和激发光能量对该反应的影响, 实验结果 见表 1 . 从实验结果我们可以发现, 在 $185 \& 254 \mathrm{~nm}$ 光照 条件下, 以四氢呋喃作溶剂时, 该反应不能进行(表 1 , Entry 1). 当以乙酸乙酯或丙酩作溶剂时, 分别得到了 $14 \%$ 和 $28 \%$ 的目标产物(表 1, Entries 2,3). 当以甲醇或 二氯甲烷作溶剂时，能够以 $31 \%$ 的产率得到目标产物 (表 1, Entries 4,5). 令我们高兴的是, 当以乙腈作溶剂 时，该反应可以以 $74 \%$ 的分离产率生成目标产物(表 1 , Entry 6). 因此, 选择乙腈作为该反应的标准溶剂. 在此

\footnotetext{
*E-mail: xinhpeng@njust.edu.cn, hao_guo@fudan.edu.cn

Received December 17, 2014; revised January 14, 2015; published online February 2, 2015.

Project supported by the Specialized Research Fund for the Doctoral Program of Higher Education (No. 20110071120004).

教育部高等学校博士学科点专项科研基金(No. 20110071120004)资助项目.
} 
基础上，我们又进一步考察了激发光能量对该反应的影 响. 尝试着降低激发光的能量, 发现当激发光波长为 $254 \mathrm{~nm}$ 时, 反应的产率降到了 $64 \%$ (表 1, Entry 7). 当激 发光波长为 $313 \mathrm{~nm}$ 时, 反应的产率降到了 $59 \%$ (表 1 , Entry 8). 进一步提高激发光波长至 $365 \mathrm{~nm}$ 时, 反应的 产率有所上升为 70\%(表 1, Entry 9), 但依然低于激发光 波长为 $185 \& 254 \mathrm{~nm}$ 时的收率(表 1 , Entry 9). 于是, 选取 $185 \& 254 \mathrm{~nm}$ 作为该反应的光照条件. 最终, 我们确定 了该反应的最佳反应条件为: 室温下以乙腈为溶剂, 在 $185 \& 254 \mathrm{~nm}$ 紫外光条件下进行.

表 1 反应条件优化 ${ }^{a}$

Table 1 Optimization of the reaction conditions

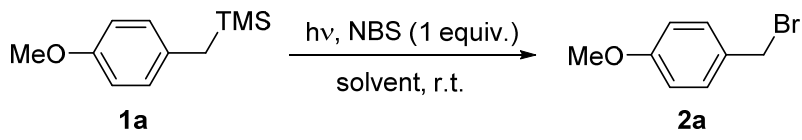

\begin{tabular}{clclc}
\hline Entry & Solvent & Wavelength/nm & Time/h & Yield $^{b} \%$ of 2a \\
\hline 1 & THF & $185 \& 254$ & 8 & $\mathrm{NR}$ \\
2 & EtOAc & $185 \& 254$ & 2.5 & 14 \\
3 & Acetone & $185 \& 254$ & 2 & 28 \\
4 & Methanol & $185 \& 254$ & 2.5 & 31 \\
5 & $\mathrm{CH}_{2} \mathrm{Cl}_{2}$ & $185 \& 254$ & 1.5 & 31 \\
6 & $\mathrm{MeCN}_{2}$ & $185 \& 254$ & 1.5 & $80(74)^{c}$ \\
7 & $\mathrm{MeCN}$ & 254 & 2 & 64 \\
8 & $\mathrm{MeCN}$ & 313 & 2 & 59 \\
9 & $\mathrm{MeCN}$ & 365 & 2 & 70 \\
\hline
\end{tabular}

${ }^{a}$ A solution of 1a $(0.2 \mathrm{mmol})$ and NBS $(0.2 \mathrm{mmol})$ in deaerated solvent $(10$ $\mathrm{mL})$ in a quartz reaction tube was irradiated by a Matrix-10 reactor with sixteen ultraviolet lamps ( $10 \mathrm{~W}$ per lamp) at r.t. under argon atmosphere. ${ }^{b}$ Yield based on ${ }^{1} \mathrm{H}$ NMR analysis of the crude reaction mixture using $\mathrm{CH}_{2} \mathrm{Br}_{2}$ as the internal standard. ${ }^{c}$ Isolated yield.

\section{2 普适性研究}

在最佳反应条件下, 我们对该反应体系的底物普适 性进行了考察, 如表 2 所示. 实验结果表明: 当苯环上 连有强给电子基团时，比如甲氧基、乙氧基、茮氧基、 苯氧基, 该反应能够顺利进行, 以中等到良好的分离产 率生成目标产物(表 2, Entries 1 4). 而当苯环上连有弱 给电子基团时，比如甲基，该反应也可以以良好的收率 得到相应的苠溴化合物(表 2, Entry 5). 当苯环上连有弱 拉电子基团时，比如氯原子，在反应底物完全消失之后， 无法分离到任何目标产物, 仅能观察到少量无法鉴定的 副产物的生成(表 2, Entry 6). 当苯环上连有强拉电子基 才时，比如羧基、酰胺基，该反应仅能得到非常复杂的 反应产物(表 2, Entries 7,8), 进一步的研究还表明: 该 反应不仅仅适用于苯基取代的底物, 对于其它稠环芳香 族化合物, 比如萗和葸, 该反应同样可以顺利进行, 目 标产物的产率可以达到良好到中等(表 2, Entries 9 11).

接下来, 我们又对其它硅基取代的苄基硅试剂的反 应性进行了考察(表 3). 实验结果表明: 三甲基硅基、二
表 $21 \mathrm{a} \sim 1 \mathrm{k}$ 的光化学反应 ${ }^{a}$

Table 2 The photoreaction of $\mathbf{1 a} \sim \mathbf{1 k}$

\begin{tabular}{|c|c|c|c|c|c|}
\hline $\mathrm{Ar}-$ & & $\frac{h v(\lambda=185 \& 254 \mathrm{~nm}), \mathrm{N}}{\mathrm{MeCN} \text {, r.t. }}$ & $\mathrm{S}(1 \mathrm{e}$ & & $\mathrm{Ar} \frac{2}{2}^{\mathrm{Br}}$ \\
\hline Entry & 1 & $\mathrm{Ar}$ & Time/h & 2 & Yield $/ \%$ of 2 \\
\hline 1 & 1a & $4-\mathrm{MeOC}_{6} \mathrm{H}_{4}$ & 1.5 & $2 \mathbf{a}$ & 74 \\
\hline 2 & 1b & 4-EtOC ${ }_{6} \mathrm{H}_{4}$ & 1.5 & $2 b$ & 82 \\
\hline 3 & 1c & 4- $\mathrm{PhCH}_{2} \mathrm{OC}_{6} \mathrm{H}_{4}$ & 1.5 & $2 c$ & 63 \\
\hline 4 & 1d & $4-\mathrm{PhOC}_{6} \mathrm{H}_{4}$ & 2 & 2d & 63 \\
\hline 5 & 1e & $2,3,4,5,6-\mathrm{MeC}_{6}$ & 1.5 & $2 e$ & 83 \\
\hline 6 & 1f & $4-\mathrm{ClC}_{6} \mathrm{H}_{4}$ & 3 & $2 f$ & Trace $^{c}$ \\
\hline 7 & $1 g$ & $4-\mathrm{HOOCC}_{6} \mathrm{H}_{4}$ & 4 & $2 g$ & Complicated \\
\hline 8 & $1 \mathrm{~h}$ & $4-\left[(i-\mathrm{Pr})_{2} \mathrm{~N}(\mathrm{O}) \mathrm{C}\right] \mathrm{C}_{6} \mathrm{H}_{4}$ & 2 & $2 \mathrm{~h}$ & Complicated \\
\hline 9 & $\mathbf{1 i}$ & 1-Naphthyl & 8 & $2 \mathbf{i}$ & 72 \\
\hline 10 & $\mathbf{1 j}$ & 2-Naphthyl & 4 & $2 \mathbf{j}$ & 63 \\
\hline 11 & $1 k$ & 9-Anthracenyl & 4 & $2 \mathbf{k}$ & 86 \\
\hline
\end{tabular}

${ }^{a}$ A solution of $1(0.2 \mathrm{mmol})$ and NBS $(0.2 \mathrm{mmol})$ in deaerated $\mathrm{MeCN}(10 \mathrm{~mL})$ in a quartz reaction tube was irradiated by a Matrix 185-10 reactor with sixteen $185 \& 254 \mathrm{~nm}$ ultraviolet lamps ( $10 \mathrm{~W}$ per lamp) at r.t. under argon atmosphere. ${ }^{b}$ Isolated yield. ${ }^{c}$ Some unidentified products were formed.

甲基乙基硅基、三乙基硅基、二甲基苯基硅基取代的苄 基硅试剂都可以顺利进行此反应，以良好的产率获得目 标产物。

表 $31 \mathrm{a}$ 和 $1 \mathrm{l} \sim \mathbf{1 n}$ 的光化学反应 ${ }^{a}$

Table 3 The photoreaction of $1 \mathrm{a}$ and $\mathbf{1 l} \sim \mathbf{1 n}$

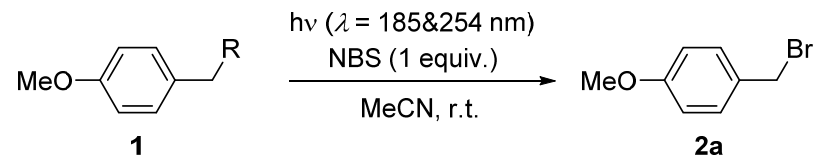

\begin{tabular}{cclcc}
\hline Entry & $\mathbf{1}$ & \multicolumn{1}{c}{$\mathrm{R}$} & Time/h & Yield $^{b} / \%$ of $\mathbf{2 a}$ \\
\hline 1 & $\mathbf{1 a}$ & $\mathrm{SiMe}_{3}$ & 1.5 & 74 \\
2 & $\mathbf{1 l}$ & $\mathrm{SiEtMe}_{2}$ & 1.5 & 71 \\
3 & $\mathbf{1 m}$ & $\mathrm{SiEt}_{3}$ & 1.5 & 80 \\
4 & $\mathbf{1 n}$ & $\mathrm{SiPhMe}_{2}$ & 1.5 & 70 \\
\hline
\end{tabular}

${ }^{a}$ A solution of $\mathbf{1}(0.2 \mathrm{mmol})$ and $\mathrm{NBS}(0.2 \mathrm{mmol})$ in deaerated $\mathrm{MeCN}(10 \mathrm{~mL})$ in a quartz reaction tube was irradiated by a Matrix 185-10 reactor with sixteen $185 \& 254 \mathrm{~nm}$ ultraviolet lamps ( $10 \mathrm{~W}$ per lamp) at r.t. under argon atmosphere. ${ }^{b}$ Isolated yield.

另外，值得说明的是：在上述底物普适性的研究过 程中, 没有观测到任何二溴产物的生成.

\section{3 机理研究}

根据上述实验结果及相关文献报道 ${ }^{[16 ~ 21]}$, 我们提 出了该反应可能的机理(Scheme 1). 首先, 在紫外光照 条件下, $N$-溴代丁二酰亚胺的氮一溴键发生均裂生成了 溴自由基 ${ }^{[16,17]}$, 溴自由基与茮基三甲基硅烷(1)发生了 单电子转移(SET)过程生成了中间体 $\mathbf{3}^{[18]}$, 而中间体 $\mathbf{3}$ 在 乙腈溶剂的作用下快速地发生脱硅反应，从而生成茮基 自由基 $4^{[19]}$. 同时, 溶液中的溴负离子与硅烷正离子结 合生成了溴硅烷 $\mathbf{5}$. 在紫外光照条件下，溴硅烷 $\mathbf{5}$ 的溴一 
硅键发生均裂再次生成溴自由基. 最后苄基自由基 $\mathbf{4}$ 与 溴自由基发生偶联反应, 从而生成了最终产物 2. 在这 里, 溴原子起到了两个作用. 首先, 它是促进碳一硅键断 裂的单电子转移(SET)的媒介. 其次, 它是生成最终产 物的溴来源. 在整个过程中, 光照条件下由氮-溴键和 硅一溴键发生均裂从而得到溴原子是非常重要的关键步 骤.

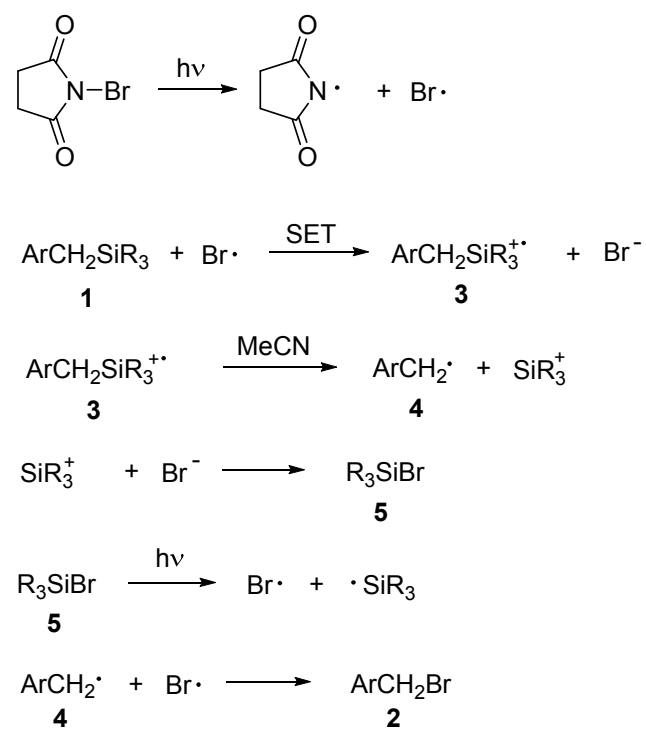

图式 1 可能的反应机理

Scheme 1 The possible reaction mechanism

\section{2 结论}

发展了一套光引发的有机硅试剂通过单电子转移 脱硅随后发生溴代反应，从而高效合成茮溴类化合物的 方法. 通过对该反应细致的研究我们提出了反应的可能 机理. 在整个反应过程中, 溴原子起了两次关键的作用. 第一次是作为单电子转移的媒介, 促使碳一硅键的高效 断裂; 第二次是作为溴源, 从而最终完成向茮溴产品的 转化. 光照是实现氮一溴键和硅一溴键均裂, 从而生成溴 原子的必要条件. 本研究丰富了溴代反应的有机合成方 法学, 为苄溴类化合物的合成提供了一条新的途径.

\section{3 实验部分}

\section{1 仪器与试剂}

所有光化学反应均在 Matrix-10 型光反应器中进行; ${ }^{1} \mathrm{H}$ NMR (400 MHz) 和 ${ }^{13} \mathrm{C}$ NMR (100 MHz)检测采用 AVANCE III 400 核磁共振仪; 红外检测采用 AVATAR360 型(FT-IR)红外光谱仪; HRMS (ESI)检测采 用 APEX III ${ }^{\mathrm{TM}}$ ESI-FTICRMS 质谱仪; HRMS (EI)检测采 用 Water GCT CA176 质谱仪; 熔点检测采用 WRS-2 熔 点仪. 无水溶剂均由实验室蒸馏所制; 化合物 $\mathbf{1 a}^{[20]}$, $\mathbf{1} \mathbf{d}^{[21]}, \mathbf{1} \mathbf{e}^{[22]}, \mathbf{1 f}^{[23]}, \mathbf{1 g}^{[24]}, \mathbf{1 h}^{[25]}, \mathbf{1 i}^{[26]}, \mathbf{1 j}^{[27]}, \mathbf{1 k}^{[28]}$ ，根据相
关文献自制; 其它试剂药品全部从市场直接购买.

\section{2 实验方法}

\section{2 .1 原料的合成}

以 $1 \mathrm{~b}$ 为例, 在无水无氧, 氩气保护条件下，向装有 磁子的 $50 \mathrm{~mL}$ 圆底烧瓶中依次加入镁条 $(250 \mathrm{mg}, 10.4$ $\mathrm{mmol}) 、$ 无水四氢呋喃 $(5 \mathrm{~mL})$ 、三甲基氯硅烷 $(2 \mathrm{~mL}, 16.0$ $\mathrm{mmol}$ )和一粒磑. 室温下摚拌 $15 \mathrm{~min}$ 后, 逐滴加入溶于 $6 \mathrm{~mL}$ 无水四氢呋喃的 4-乙氧基芐氯 $(990 \mathrm{mg}, 5.8 \mathrm{mmol}$ ), 室温下搅拌 $12 \mathrm{~h}$. 反应结束后, 加入 $10 \mathrm{~mL}$ 饱和氯化铵 溶液淬灭反应，乙酸乙酯萃取 $(10 \mathrm{~mL} \times 3)$, 无水硫酸镁 干燥. 干燥后的有机相脱出溶剂, 经快速柱层析分离得 到 $752 \mathrm{mg}$ 化合物 1b, 产率 $62 \%$. 化合物 $1 \mathrm{c}$ 按类似方法 合成.

4-乙氧基苄基三甲基硅烷 (1b): 无色液体, 产率 62\%. ${ }^{1} \mathrm{H}$ NMR (400 MHz, $\left.\mathrm{CDCl}_{3}\right) \delta: 6.96(\mathrm{~d}, J=8.4 \mathrm{~Hz}$, $2 \mathrm{H}), 6.83(\mathrm{~d}, J=8.4 \mathrm{~Hz}, 2 \mathrm{H}), 4.04$ (q, $J=7.2 \mathrm{~Hz}, 2 \mathrm{H}), 2.07$ (s, 2H), 1.45 (t, $J=7.2 \mathrm{~Hz}, 3 \mathrm{H}), 0.05(\mathrm{~s}, 9 \mathrm{H}) ;{ }^{13} \mathrm{C} \mathrm{NMR} \delta$ : $155.8,132.1,128.7,114.2,63.2,25.6,14.9,-2.0$; IR (neat) $v: 1613,1577,1510,1479 \mathrm{~cm}^{-1}$; HRMS (ESI) calcd for $\mathrm{C}_{12} \mathrm{H}_{21} \mathrm{OSi}\left(\mathrm{M}+\mathrm{H}^{+}\right)$209.1356, found 209.1352.

4- 芐氧基苄基三甲基硅烷(1c)：白色固体，产率 $83 \%$. m.p. $67.1 \sim 67.6{ }^{\circ} \mathrm{C} ;{ }^{1} \mathrm{H}$ NMR $\left(400 \mathrm{MHz}, \mathrm{CDCl}_{3}\right) \delta$ : $7.46 \sim 7.31(\mathrm{~m}, 5 \mathrm{H}), 6.93(\mathrm{~d}, J=8.4 \mathrm{~Hz}, 2 \mathrm{H}), 6.86(\mathrm{~d}, J=$ $8.4 \mathrm{~Hz}, 2 \mathrm{H}) 5.03(\mathrm{~s}, 2 \mathrm{H}), 2.02(\mathrm{~s}, 2 \mathrm{H}),-0.01(\mathrm{~s}, 9 \mathrm{H}) ;{ }^{13} \mathrm{C}$ NMR $\delta$ : 155.8, 137.4, 132.7, 128.8, 128.5, 127.8, 127.5, 114.6, 70.1, 25.7, -2.0; IR (KBr) v: 1605, 1576, 1514, $1448,1407 \mathrm{~cm}^{-1}$; HRMS (ESI) calcd for $\mathrm{C}_{17} \mathrm{H}_{23} \mathrm{OSi}(\mathrm{M}+$ $\mathrm{H}^{+}$) 271.1513, found 271.1516.

4-甲氧基芐基乙基二甲基硅烷(11): 在无水无氧，氩 气保护条件下，向装有磁子的 $50 \mathrm{~mL}$ 圆底烧瓶中依次加 入镁条 $(150 \mathrm{mg}, 6.3 \mathrm{mmol}) 、$ 无水四氢呋喃 $(10 \mathrm{~mL}) 、 乙$ 基二甲基氯硅烷 $(1.4 \mathrm{~mL}, 10.0 \mathrm{mmol}$ )和一粒碘. 室温下 搅拌 $15 \mathrm{~min}$ 后, 逐滴加入溶于 $10 \mathrm{~mL}$ 无水四氢呋喃的 4-甲氧基苄氯 $(678 \mu \mathrm{L}, 5.0 \mathrm{mmol})$, 室温下搅拌 $12 \mathrm{~h}$. 反 应结束后, 加入 $10 \mathrm{~mL}$ 饱和氯化铵溶液淬灭反应, 乙酸 乙酯萃取 $(10 \mathrm{~mL} \times 3)$, 无水硫酸镁干燥. 干燥后的有机 相脱出溶剂, 经快速柱层析分离得到 $697 \mathrm{mg}$ 化合物 11, 无色液体, 产率 $67 \% .{ }^{1} \mathrm{H}$ NMR $\left(400 \mathrm{MHz}, \mathrm{CDCl}_{3}\right) \delta: 6.92$ (d, $J=8.4 \mathrm{~Hz}, 2 \mathrm{H}), 6.78$ (d, $J=8.4 \mathrm{~Hz}, 2 \mathrm{H}), 3.78$ (s, 3H), $2.01(\mathrm{~s}, 2 \mathrm{H}), 0.93(\mathrm{t}, J=8.0 \mathrm{~Hz}, 3 \mathrm{H}), 0.48(\mathrm{q}, J=8.0 \mathrm{~Hz}$, $2 \mathrm{H}),-0.05(\mathrm{~s}, 6 \mathrm{H}) ;{ }^{13} \mathrm{C}$ NMR $\delta$ : $156.5,132.3,128.8$, 113.4, 55.2, 23.6, 7.2, 6.4, -4.4; IR (neat) $v: 1614,1585$, $1508,1469 \mathrm{~cm}^{-1}$; HRMS (ESI) calcd for $\mathrm{C}_{12} \mathrm{H}_{21} \mathrm{OSi}(\mathrm{M}+$ $\mathrm{H}^{+}$) 209.1356, found 209.1356.

4-甲氧基苄基三乙基硅烷(1 m): 在无水无氧, 氩气 
保护条件下，向装有磁子的 $50 \mathrm{~mL}$ 圆底烧瓶中依次加入 镁条 $(152 \mathrm{mg}, 6.3 \mathrm{mmol}) 、$ 无水四氢呋喃 $(20 \mathrm{~mL}) 、 三 乙$ 基氯硅烷 $(1.7 \mathrm{~mL}, 10.0 \mathrm{mmol})$ 和一粒碘. 室温下搅拌 15 $\min$ 后, 逐滴加入溶于 $5 \mathrm{~mL}$ 无水四氢呋喃的 4-甲氧基苄 氯(678 $\mu \mathrm{L}, 5.0 \mathrm{mmol})$, 室温下搅拌 $19 \mathrm{~h}$. 反应结束后, 加入 $10 \mathrm{~mL}$ 饱和氯化铵溶液淬灭反应, 乙酸乙酯萃取 $(10 \mathrm{~mL} \times 3)$, 无水硫酸镁干燥. 干燥后的有机相脱出溶 剂, 经快速柱层析分离得到 $710 \mathrm{mg}$ 化合物 $\mathbf{1 m}$, 无色液 体, 产率 60\%. ${ }^{1} \mathrm{H}$ NMR $\left(400 \mathrm{MHz}, \mathrm{CDCl}_{3}\right) \delta: 6.93$ (d, $J=$ $8.4 \mathrm{~Hz}, 2 \mathrm{H}), 6.76$ (d, $J=8.4 \mathrm{~Hz}, 2 \mathrm{H}), 3.76$ (s, 3H), 2.02 (s, $2 \mathrm{H}), 0.91$ (t, $J=8.0 \mathrm{~Hz}, 9 \mathrm{H}), 0.49$ (q, $J=8.0 \mathrm{~Hz}, 6 \mathrm{H}) ;{ }^{13} \mathrm{C}$ NMR $\delta$ : 156.4, 132.4, 128.8, 113.6, 55.2, 20.2, 7.3, 2.9; IR (neat) $v: 1617,1582,1511,1466 \mathrm{~cm}^{-1}$; HRMS (ESI) calcd for $\mathrm{C}_{14} \mathrm{H}_{25} \mathrm{OSi}\left(\mathrm{M}+\mathrm{H}^{+}\right)$237.1669, found 237.1663.

4-甲氧基茮基苯基二甲基硅烷(1n): 在无水无氧, 氩气保护条件下, 向装有磁子的 $50 \mathrm{~mL}$ 圆底烧瓶中依次 加入镁条 $(155 \mathrm{mg}, 6.5 \mathrm{mmol})$ 、无水四氢呋喃 $(20 \mathrm{~mL})$ 、 苯基二甲基氯硅烷 $(1.7 \mathrm{~mL}, 10.0 \mathrm{mmol})$ 和一粒碘. 室温 下搅拌 $15 \mathrm{~min}$ 后, 逐滴加入溶于 $5 \mathrm{~mL}$ 无水四氢呋喃的 4-甲氧基苄氯(678 $\mu \mathrm{L}, 5.0 \mathrm{mmol})$, 室温下搅拌 $12 \mathrm{~h}$. 反 应结束后, 加入 $20 \mathrm{~mL}$ 饱和氯化铵溶液淬灭反应, 乙酸 乙酯萃取 $(20 \mathrm{~mL} \times 3)$, 无水硫酸镁干燥. 干燥后的有机 相脱出溶剂, 经快速柱层析分离得到 $953 \mathrm{mg}$ 化合物 $\mathbf{1 n}$, 无色液体, 产率 $74 \% .{ }^{1} \mathrm{H}$ NMR $\left(400 \mathrm{MHz} \mathrm{CDCl}_{3}\right) \delta$ : $7.47 \sim 7.43(\mathrm{~m}, 2 \mathrm{H}), 7.37 \sim 7.32(\mathrm{~m}, 3 \mathrm{H}), 6.84(\mathrm{~d}, J=8.4$ $\mathrm{Hz}, 2 \mathrm{H}), 6.74$ (d, J=8.4 Hz, 2H), 3.76 (s, 3H), 2.23 (s, 2H), 0.23 (s, $6 \mathrm{H}) ;{ }^{13} \mathrm{C}$ NMR $\delta$ : 156.6, 138.6, 133.7, 131.5, 129.1, 129.0, 127.7, 113.6, 55.2, 24.8, -3.5; IR (neat) $v$ : 1614, 1582, 1508, 1463, 1439, $1425 \mathrm{~cm}^{-1}$; HRMS (EI) calcd for $\mathrm{C}_{16} \mathrm{H}_{20} \mathrm{OSi} 256.1283$, found 256.1282.

\section{2 .2 光反应标准方法}

在无水无氧, 氩气保护条件下, 向 $10 \mathrm{~mL}$ 石英反应 管依次加入 $N$-溴代丁二酰亚胺 $(0.2 \mathrm{mmol})$ 、茮基三甲基 硅烷 1 (0.2 mmol)、无水乙腈 $(10 \mathrm{~mL})$, 之后经氩气超声 脱气 $10 \mathrm{~min}$. 在 Matrix185-10 光化学反应器中室温条件 下反应至 TLC 监测反应结束. 反应液脱出大部分溶剂 后, 用饱和碳酸氢钠溶液洗涤, 乙醚萃取, 无水硫酸钠 干燥. 干燥后的有机相经快速柱层析纯化得到产物 2.

4- 甲氧基苄溴 $(2 a){ }^{[29]}$ : 黄色液体，产率 $74 \%$. ${ }^{1} \mathrm{H}$ NMR $\left(\mathrm{CDCl}_{3}, 400 \mathrm{MHz}\right) \delta: 7.30(\mathrm{~d}, J=8.8 \mathrm{~Hz}, 2 \mathrm{H}), 6.85$ (d, $J=8.8 \mathrm{~Hz}, 2 \mathrm{H}), 4.48$ (s, 2H), 3.78 (s, 3H).

4-乙氧基茮溴 $(2 \mathrm{~b})$ : 黄色液体，产率 $82 \% .{ }^{1} \mathrm{H} \mathrm{NMR}$ $\left(\mathrm{CDCl}_{3}, 400 \mathrm{MHz}\right) \delta: 7.35 \sim 7.30(\mathrm{~m}, 2 \mathrm{H}), 6.89 \sim 6.85(\mathrm{~m}$, 2H), 4.52 (s, 2H), 4.03 (q, $J=7.2 \mathrm{~Hz}, 2 \mathrm{H}), 1.44$ (t, $J=7.2$ $\mathrm{Hz}, 3 \mathrm{H}) ;{ }^{13} \mathrm{C} \mathrm{NMR}\left(\mathrm{CDCl}_{3}, 100 \mathrm{MHz}\right) \delta: 159.0,130.3$,
129.6, 114.6, 63.3, 34.0, 14.7; IR (neat) v: 1712, 1611, 1511, $1472 \mathrm{~cm}^{-1}$; HRMS (EI) calcd for $\mathrm{C}_{9} \mathrm{H}_{11} \mathrm{BrO}$ 213.9993, found 213.9992.

4-苄氧基溴苠 $(2 \mathrm{c})^{[30]}$ : 淡黄色固体，产率 $63 \%$. ${ }^{1} \mathrm{H}$ NMR $\left(\mathrm{CDCl}_{3}, 400 \mathrm{MHz}\right) \delta: 7.46 \sim 7.31(\mathrm{~m}, 7 \mathrm{H}), 6.96 \sim$ $6.92(\mathrm{~m}, 2 \mathrm{H}), 5.07$ (s, 2H), $4.51(\mathrm{~s}, 2 \mathrm{H})$.

4-苯氧基苠溴 $(2 \mathrm{~d})^{[31]}$ : 淡黄色液体, 产率 $66 \% .{ }^{1} \mathrm{H}$ NMR $\left(\mathrm{CDCl}_{3}, 400 \mathrm{MHz}\right) \delta: 7.38 \sim 7.32(\mathrm{~m}, 4 \mathrm{H}), 7.16 \sim$ $7.10(\mathrm{~m}, 1 \mathrm{H}), 7.05 \sim 6.98(\mathrm{~m}, 2 \mathrm{H}), 6.98 \sim 6.93(\mathrm{~m}, 2 \mathrm{H})$, $4.51(\mathrm{~s}, 2 \mathrm{H})$.

五甲基苠溴 $(2 \mathrm{e})^{[32]}$ : 白色固体，产率 $83 \% .{ }^{1} \mathrm{H} \mathrm{NMR}$ $\left(\mathrm{CDCl}_{3}, 400 \mathrm{MHz}\right) \delta: 4.66(\mathrm{~s}, 2 \mathrm{H}), 2.34(\mathrm{~s}, 6 \mathrm{H}), 2.24 \sim$ $2.22(\mathrm{~m}, 9 \mathrm{H})$.

1-溴甲基萗 $(2 \mathbf{i})^{[33]}$ : 白色固体, 产率 $72 \% .{ }^{1} \mathrm{H} \mathrm{NMR}$ $\left(\mathrm{CDCl}_{3}, 400 \mathrm{MHz}\right) \delta: 8.16(\mathrm{~d}, J=8.8 \mathrm{~Hz}, 1 \mathrm{H}), 7.90 \sim 7.80$ $(\mathrm{m}, 2 \mathrm{H}), 7.66 \sim 7.58(\mathrm{~m}, 1 \mathrm{H}), 7.57 \sim 7.46(\mathrm{~m}, 2 \mathrm{H}), 7.45 \sim$ $7.36(\mathrm{~m}, 1 \mathrm{H}), 4.97(\mathrm{~s}, 2 \mathrm{H})$.

2-溴甲基萗 $(2 \mathbf{j})^{[34]}$ : 浅棕色固体，产率 $63 \%$. ${ }^{1} \mathrm{H}$ NMR $\left(\mathrm{CDCl}_{3}, 400 \mathrm{MHz}\right) \delta: 7.89 \sim 7.79(\mathrm{~m}, 4 \mathrm{H}), 7.57 \sim$ 7.47 (m, 3H), 4.69 (s, 2H).

9-溴甲基葱 $(2 \mathbf{k})^{[35]}$ : 黄色固体，产率 $86 \%$. ${ }^{1} \mathrm{H} \mathrm{NMR}$ $\left(\mathrm{CDCl}_{3}, 400 \mathrm{MHz}\right) \delta: 8.49(\mathrm{~s}, 1 \mathrm{H}), 8.30(\mathrm{~d}, J=8.8 \mathrm{~Hz}$, $2 \mathrm{H}), 8.04$ (d, $J=8.4 \mathrm{~Hz}, 2 \mathrm{H}), 7.64$ (t, $J=7.6 \mathrm{~Hz}, 2 \mathrm{H}), 7.50$ $(\mathrm{t}, J=7.6 \mathrm{~Hz}, 2 \mathrm{H}), 5.54(\mathrm{~s}, 2 \mathrm{H})$.

辅助材料(Supporting Information) 所合成目标化合 物的 NMR 图谱. 这些材料可以免费从本刊网站 (http://sioc-journal.cn/)上下载.

\section{References}

[1] Klein, S. M.; Zhang, C.; Jiang, Y. L. Tetrahedron Lett. 2008, 49, 2638.

[2] Stefely, J. A.; Palchaudhuri, R.; Miller, P. A.; Peterson, R. J.; Monraski, G. C.; Hergenrother, P. J.; Miller, M. J. J. Med. Chem. 2010, $53,3389$.

[3] Firouzabadi, H.; Iranpoor, N.; Ebrahimzadeh, F. Tetrahedron Lett. 2006, 47, 1771.

[4] Manna, S.; Falck, J. R. Synth. Commun. 1985, 15, 663.

[5] González-Bobes, F.; Fu, G. C. J. Am. Chem. Soc. 2006, 128, 5360.

[6] Kamal, A.; Chouhan, G. Tetrahedron Lett. 2005, 46, 1489.

[7] Tongkate, P.; Pluempanupat, W.; Chavasiri, W. Tetrahedron Lett. 2008, 49, 1146.

[8] (a) Joseph, K. M.; Larraza-Sanchez, I. Tetrahedron Lett. 2011, 52, 13.

(b) Liu, T. L.; Zhou, Y. B. Chin. J. Org. Chem. 2000, 20, 758 (in Chinese).

(刘天麟, 周懿波, 有机化学, 2000, 20, 758.)

(c) Wang, N.; Li, W.; Zhang, L.; Gao, Y.; Ji, C.; Chen, Y.; Chai, X.; Sun, H.; Bi, Y.; Wu, Q.; Meng, Q. Chin. J. Org. Chem. 2012, 32, 922 (in Chinese).

(王楠, 李文娟, 张雷, 高一军, 冀春梅, 陈莹, 柴晓云, 孙海军, 
毕毅, 吴从业, 孟庆国, 有机化学, 2012,32,922.)

[9] Larock, R. C. Comprehensive Organic Transformations: A Guide to Functional Group Preparations, 2nd ed., Wiley-VCH, New York, 1999.

[10] Cantillo, D.; Frutos, O.; Rincon, J. A.; Mateos, C.; Kappe, C. O. J. Org. Chem. 2014, 79, 223.

[11] (a) Podgoršek, A.; Stavber, S.; Zupan, M.; Iskra, J. Tetrahedron 2009, 65, 4429.

(b) Sheng, C. Q.; Li, Z. F.; Yang, C. J.; Lai, G. Q.; Qiu, H. Y. Chin. J. Org. Chem. 2008, 28, 515 (in Chinese).

(盛春荠, 李志芳, 杨成军, 来国桥, 邱化玉, 有机化学, 2008, 28,515 .)

[12] Gawande, M. B.; Deshpande, S. S.; Satam, J. R.; Jayaram, R. V. Catal. Commun. 2007, 8, 576.

[13] Baciocchi, E.; Crescenzi, M. Angew. Chem., Int. Ed. Engl. 1990, 29, 658.

[14] Bordeau, M.; Villeneuve, P.; Bennetau, B.; Dunoguès, J. J. Organomet. Chem. 1987, 331, 169.

[15] Sun, J.; Wang, Y.; Han, L.; Xu, D.; Chen, Y.; Peng, X.; Guo, H. Org. Chem. Front. 2014, 1, 1201.

[16] Day, J. C.; Govindaraj, N.; Mcbain, D. S.; Skell, P. S.; Tanko, J. M. J. Org. Chem. 1986, 51, 4959.

[17] Lüning, U.; Skell, P. S. Tetrahedron 1985, 41, 4289.

[18] Baciocchi, E.; Crescenzi, M.; Giacco, T. D. J. Chem. Soc., Perkin Trans. 1 1991, 3377.

[19] Tamai, T.; Mizuno, K.; Hashida, I.; Otsuji, Y. Bull. Chem. Soc. Jpn. 1993, 66, 3747.

[20] Coughlin, D. J.; Salomon, R. G. J. Org. Chem. 1979, 44, 3784.

[21] Ishibashi, H.; Nakatani, H.; Umei, Y.; Yamamoto, W.; Ikeda, M. J.
Chem. Soc., Perkin Trans. 1 1987, 589.

[22] Maruyama, T.; Mizuno, Y.; Shimizu, I.; Suga, S.; Yoshida, J. J. Am. Chem. Soc. 2007, 129, 1902.

[23] Protti, S.; Ravelli, D.; Mannucci, B.; Albini, A.; Fagnoni, M. Angew. Chem., Int. Ed. 2012, 51, 8577.

[24] Stern, A. J.; Swenton, J. S. J. Org. Chem. 1989, 54, 2953.

[25] Das, M.; O’Shea, D. F. Tetrahedron 2013, 69, 6448.

[26] Tatsuya, S.; Yoshihiro, M.; Susumu, K.; Naoki, K. Chem. Lett. 1985, 463.

[27] Huckins, J. R.; Rychnovsky, S. D. J. Org. Chem. 2003, 68, 10135.

[28] Bailey, S. I.; Colgan, D.; Engelhardt, L. M.; Leung, W.; Papasergion, R. I.; Raston, C. L.; White, A. H. J. Chem. Soc., Dalton Trans. 1986, 603 .

[29] Khartulyari, A. S.; Kapur, M.; Maier, M. E. Org. Lett. 2006, 8, 5833.

[30] Flaherty, D. P.; Kiyota, T.; Dong, Y.; Ikezu, T.; Vennerstrom, J. L. J. Med. Chem. 2010, 53, 7922.

[31] Christiansen, E.; Due-Hansen, M. E.; Urban, C.; Merten, N.; Pfleiderer, M.; Karlsen, K. K.; Rasmussen, S. S.; Steensgaard, M.; Hamacher, A.; Schmidt, J.; Drewke, C.; Petersen, R. K.; Kristiansen, K.; Ullrich, S.; Kostenis, E.; Kassack, M. U.; Ulven, T. ACS Med. Chem. Lett. 2010, 1, 345.

[32] Seklne, Y.; Boekelheide, V. J. Am. Chem. Soc. 1981, 103, 1777.

[33] Cui, X.; Guan, Y.; Li, N.; Lv, H.; Fu, L.; Guo, K.; Fan, X. Tetrahedron Lett. 2014, 55, 90.

[34] Suarez, D.; Laval, G.; Tu, S.; Jiang, D.; Robinson, C. L.; Scott, R.; Golding, B. T. Synthesis 2009, 1807.

[35] Shu, C.; Chen, C.-B.; Chen, W.-X.; Ye, L.-W. Org. Lett. 2013, 12, 5542.

(Li, L.; Fan, Y.) 